\title{
ON A LEMMA OF L. LORCH AND D. J. NEWMAN \\ FRED USTINA
}

(Received 25 May 1970; revised 4 July 1971)

Communicated by E. Strezelcki

\section{Introduction}

In [6], Lorch and Newman proved the following lemma:

If $g(u)$ is continuous and of bounded variation, $0 \leqq u \leqq 1$, then

$$
\int_{1}^{m}\left|\int_{0}^{1} \frac{\sin s u}{s} d g(u)\right| d s=o(\log m), \quad m \rightarrow \infty .
$$

This was extended more recently by Leviatan and Lorch ([5], Lemma 3 ) to functions which are of bounded variation on the positive real axis, where now the upper limit of integration on the inner integral is infinite.

In this paper, the two dimensional analogues of these lemmas are established. As well, an inequality of some interest in itself is obtained:

THEOREM 1. For all non-negative $a$ and $b$, finite or infinite, the following inequality holds:

$$
\begin{aligned}
& \int_{-a,-b}^{a, b}\left|\int_{-\infty,-\infty}^{\infty, \infty} \frac{\sin s u}{s} \frac{\sin t v}{t} d g(u, v)\right|^{2} d s d t \\
& \quad \leqq \int_{-a-b}\left|\int_{-\infty,-\infty} e^{-i(s u+t v)} d g(u, v)\right|^{2} d s d t .
\end{aligned}
$$

THEOREM 2. Suppose that $g(u, v)$ is of bounded variation in the Vitali sense in the real plane. Then

$$
\begin{array}{r}
\int_{1,1}^{m, n}\left|\int_{-\infty,-\infty}^{\infty, \infty} \frac{e^{-i(s u+t v)}}{s t} d g(u, v)\right| d s d t \\
=o(\log m \log n), \quad m, n \rightarrow \infty
\end{array}
$$

if and only if $d g(u, v)$ has no mass points; and

$$
\int_{1,1}^{m, n}\left|\int_{-\infty,-\infty}^{\infty, \infty} \frac{\sin s u}{s} \frac{\sin t v}{t} d g(u, v)\right| d s d t
$$




$$
=o(\log m \log n), \quad m, n \rightarrow \infty
$$

if and only if $d g(u, v)$ has no mass points bounded away from the coordinate axes.

It is easily seen that if $d g(u, v)$ has mass points along the coordinate axes, these will not contribute anything to the estimate (4). Note also, that continuity of the function $g(u, v)$ is not required for (3) and (4) to hold.

The two dimensional analogue of the lemma proved in [5], as well as of the one proved in [6], then follows immediately from (4) by a suitable restriction on the function $g(u, v)$.

\section{Some Preliminary Results}

Definitions of bounded variation for functions of two variables are given by Clarkson and Adams [3]. The proofs will be given under the assumption that $g(u, v)$ is of bounded variation in the Hardy-Krause sense. That the results also hold for functions which are of bounded variation in the Vitali sense follows the observation that if $g(u, v)$ is of bounded variation in the Vitali sense, it is always possible to construct another function, $h(u, v)$, of bounded variation in the Hardy-Krause sense, such that the measures $d g(u, v)$ and $d h(u, v)$ are equal. The difference between the positive and negative variation functions of $g(u, v)$ is one such function.

We denote the rectangle whose vertices are at $\left(u^{\prime \prime}, v^{\prime \prime}\right),\left(u^{\prime \prime}, v^{\prime}\right),\left(u^{\prime}, v^{\prime \prime}\right)$ and $\left(u^{\prime}, v^{\prime}\right), u^{\prime \prime}>u^{\prime}, v^{\prime \prime}>v^{\prime}$, by $\left[u^{\prime \prime}, v^{\prime \prime} ; u^{\prime}, v^{\prime}\right]$, and let $g\left(u^{\prime \prime}, v^{\prime \prime} ; u^{\prime}, v^{\prime}\right)$ denote the difference $g\left(u^{\prime \prime}, v^{\prime \prime}\right)-g\left(u^{\prime \prime}, v^{\prime}\right)-g\left(u^{\prime}, v^{\prime \prime}\right)+g\left(u^{\prime}, v^{\prime}\right)$.

If $g(u, v)$ is of bounded variation in the sense of Hardy-Krause in the real plane, then by direct analogy with the one dimensional case [(9], ch. XVI), its modified Fourier-Stieltjes transform, or the modified transform of $d g(u, v)$, is given by

$$
\phi(s, t)=\frac{1}{2 \pi} \int_{-\infty,-\infty}^{\infty, \infty} e^{-i(s u+t v)} d g(u, v) .
$$

$\phi(s, t)$ exists and is bounded uniformly for all $s, t$.

LEMMA 1. If $g(u, v)$ is of bounded variation, then for arbitrary, fixed real numbers $p$ and $q, g(u+p, v+q ; u-p, v-q)$ is absolutely and square integrable in the real plane.

Proof. It is sufficient to prove the lemma for $p$ and $q$ positive. Also, since $g(u, v)$ is of bounded variation, and so may be expressed as the difference between its positive and negative variation functions which are bounded, non-negative and positively monotonic $([7]$, p. 250$)$, the problem is reduced to proving the 
lemma under the assumption that $g(u, v)$ is already positively monotonic, that is, for positive $p$ and $q, g(u+p, v+q ; u-p, v-q) \geqq 0$. Now let

$$
\begin{gathered}
f(a, b)=\int_{-a,-b}^{a, b} g(u+p, v+q ; u-p, v-q) d u d v \\
=\left\{\int_{a-p, b-q}^{a+q, b+p}-\int_{-a-p, b-q}^{-a+p, b+q}-\int_{a-p,-b-q}^{a+p,-b+q}+\int_{-a-p,-b-q}^{-a+p,-b+q}\right\} g(u, v) d u d v
\end{gathered}
$$

Let $a, b \rightarrow \infty$. Then $g(u, v)$ tends to definite limits which we denote by $g(\infty, \infty), g(-\infty, \infty), g(\infty,-\infty)$ and $g(-\infty,-\infty)$ in the respective regions of integration, so that

$$
\begin{aligned}
\lim f(a, b) & =4 p q g(\infty, \infty ;-\infty,-\infty) \\
& =4 p q V(g), a, b \rightarrow \infty,
\end{aligned}
$$

where $V(g)$ is the total variation of $g(u, v)$. This proves the absolute integrability. That $g(u+p, v+q ; u-p, v-q)$ is also square integrable follows the observation that it is of bounded variation, hence bounded in the real plane.

Lemma 2. If $g(u, v)$ is of bounded variation in the real plane, then

$$
\begin{aligned}
& \int_{-\infty,-\infty}^{\infty, \infty}|g(u+p, v+q ; u-p, v-q)|^{2} d u d v \\
& \quad=16 \int_{-\infty,-\infty}^{\infty, \infty}|\phi(s, t)|^{2} \frac{\sin ^{2} p s}{s^{2}} \frac{\sin ^{2} q t}{t^{2}} d s d t .
\end{aligned}
$$

Proof. An elementary calculation shows that $\phi(s, t)\left\{e^{i p s}-e^{-i p s}\right\}\left\{e^{i q t}-e^{-i, y t}\right\}=\frac{1}{2 \pi} \int_{-\infty, \infty}^{\infty, \infty} e^{-i(s u+t v)} d g(u+p, v+q ; u-p, v-q)$. Integrating by parts ([8], p. 38), and noting that $g(u+p, v+q ; u-p, v-q) \rightarrow 0$ as $u, v \rightarrow \pm \infty$ independently, then gives the result

$$
\phi(s, t) \frac{\sin p s}{s} \frac{\sin q t}{t}=\frac{1}{2 \pi} \int_{-\infty,-\infty}^{\infty, \infty} e^{-i(s u+t v)} g(u+p, v+q ; x-o, v-q) d u d v,
$$

so that the expression on the left is the modified Fourier transform of $g(u+p, v+q ; u-p, v-q)$, a function which is absolutely ond square integrable in the real plane. The proof is completed by the application of Parseval's relation ([2], p. 67) to (5).

\section{Proof of Theorem 1}

If the integral on the right is infinite, the equality is obvious. Otherwise

(6) $\left|\int_{-\infty,-\infty}^{\infty, \infty} \frac{\sin s u}{s} \frac{\sin t v}{t} d g(u, v)\right|$ 


$$
\begin{aligned}
& =\frac{1}{4}\left|\int_{-\infty,-\infty}^{\infty, \infty}\left\{e^{i(s u+t v)}+e^{-i(s u+t v)}-e^{i(s u-t v)}-e^{-i(s u-t v)}\right\} \frac{d g(u, v)}{s t}\right| \\
& \quad \leqq \frac{\pi}{2}\left\{\left|\frac{\phi(-s,-t)}{s t}\right|+\left|\frac{\phi(s, t)}{s t}\right|+\left|\frac{\phi(-s, t)}{s t}\right|+\left|\frac{\phi(s,-t)}{s t}\right|\right\}
\end{aligned}
$$

Then, since $(a+b+c+d)^{2} \leqq 4\left(a^{2}+b^{2}+c^{2}+d^{2}\right)$,

$$
\begin{aligned}
\int_{-a,-b}^{a, b} & \left|\int_{-\infty,-\infty}^{\infty, \infty} \frac{\sin s u}{s} \frac{\sin t v}{t} d g(u, v)\right|^{2} d s d t \\
& \leqq \pi^{2}\left\{\int_{-a,-b}^{a, b} \frac{|\phi(-s,-t)|^{2}}{s^{2} t^{2}} d s d t+\int_{-a,-b}^{a, b} \frac{|\phi(s, t)|^{2}}{s^{2} t^{2}} d s d t\right. \\
& +\int_{-a,-b}^{a, b} \frac{|\phi(-s, t)|^{2}}{s^{2} t^{2}} \int_{-a,-b}^{a b} \frac{|\phi(s,-t)|^{2}}{s^{2} t^{2}} \\
& =4 \pi^{2} \int_{-a,-b}^{a, b} \frac{|\phi(s, t)|^{2}}{s^{2} t^{2}} d s d t \\
& =\int_{-a,-b}^{a, b}\left|\int_{-\infty,-\infty}^{\infty, \infty} \frac{e^{-i(s u+t v)}}{s t} d g(u, v)\right|^{2} d s d t
\end{aligned}
$$

by suitably changing the variables. This proves Theorem 1 .

REMARKS. In the one dimensional case we also have, trivially,

$$
\left|\int_{-\infty}^{\infty} \sin s u d g(u)\right| \leqq\left|\int_{-\infty}^{\infty} e^{-i s u} d g(u)\right|
$$

for every value of $s$. In the two dimensional case, it is equally obvious that

$$
\left|\int_{-\infty,-\infty}^{\infty, \infty} \sin s u \sin t v d g(u, v)\right| \leqq\left|\int_{-\infty,-\infty}^{\infty, \infty} e^{-i s u} \sin t v d g(u, v)\right|
$$

for every choice of $s$ and $t$. It is also true that if $g(u, v)$ factors, so that $g(u, v)=g^{\prime}(u) \cdot g^{\prime \prime}(v)$, then

$$
\left|\int_{-\infty,-\infty}^{\infty, \infty} \sin s u \sin t v d g(u, v)\right| \leqq\left|\int_{-\infty,-\infty}^{\infty, \infty} e^{-i(s u+t v)} d g(u, v)\right|,
$$

for then the proof of (8) reduces to the proof of the inequality (7). However, the inequality (8) does not hold in general. For let $s=t=1$, and let

$$
g(u, v)=0,\left\{\begin{array}{l}
u<0, v<0 \\
u<0, v \geqq 0 \\
u \geqq 0, v<0
\end{array}\right.
$$




$$
\begin{aligned}
& g(u, v)=1, \begin{cases}0 \leqq u<\pi / 2, & 0 \leqq v \\
0 \leqq u & 0 \leqq v<\pi / 2\end{cases} \\
& g(u, v)=2, \quad \pi / 2 \leqq u, v,
\end{aligned}
$$

so that $d g(u, v)=1$ when $(u, v)=(0,0)$ and when $(u, v)=(\pi / 2, \pi / 2)$, and $d g(u, v)=0$ otherwise. Then the left side of the inequality (8) equals 1 , and the right side equals 0 .

\section{Proof of Theorem 2}

The proof is an obvious adaptation of the proof given by Lorch and Newman in [6]. We first prove the inequality (4) and its converse.

Let $\Delta^{\prime}=[a, b ;-a,-b]$, and let $\Delta^{\prime \prime}=\sim(a, b ;-a,-b)$, where $a$ and $b$ are any positive, real numbers. Then

$$
\begin{aligned}
& I(m, n)=\int_{1,1}^{m, n}\left|\int_{-\infty,-\infty}^{\infty, \infty} \frac{\sin s u}{s} \frac{\sin t v}{t} d g(u, v)\right| d s d t \\
& \quad=\int_{1,1}^{m, n}\left\{\left|\int_{\Delta^{\prime}}\right|+\left|\int_{\Delta^{\prime \prime}}\right|\right\} d s d t \\
& =I^{\prime}(m, n)+I^{\prime \prime}(m, n) .
\end{aligned}
$$

To estimate $I^{\prime}(m, n)$, let integers $h$ and $k$ be determined by the inequalities

$$
2^{h}<m \leqq 2^{h+1}, 2^{k}<n \leqq 2^{k+1},
$$

and assume that the measure $d g(u, v)$ is identically zero on $\Delta^{\prime \prime}$. Then, applying the Cauchy-Schwarz inequality for integrals, the inequality (6), and Lemma 2 ,

$$
\begin{aligned}
& I^{\prime}(m, n)=\int_{1,1}^{m, n}\left|\int_{-a,-b}^{a, b} \frac{\sin s u}{s} \frac{\sin t v}{t} d g(u, v)\right| d s d t \\
& \leqq \sum_{0,0}^{h, k} \int_{2^{i, 2},}^{2^{t+1}, 2^{j+1}}\left|\int_{-\infty,-\infty}^{\infty, \infty} \frac{\sin s u}{s} \frac{\sin t v}{t} d g(u, v)\right| d s d t \\
& \leqq \sum_{0,0}^{h, k}\left\{2^{i+j} \int_{2^{i, 2 j}}^{2^{i+1}, 2^{j+1}}\left|\int_{-\infty,-\infty}^{\infty, \infty} \frac{\sin s u}{s} \frac{\sin t v}{t} d g(u, v)\right|^{2} d s d t\right\}^{\frac{1}{2}} \\
& \leqq \pi \sum_{0.0}^{h, k}\left\{2 ^ { i + j } \int _ { 2 ^ { i , 2 ^ { j } } } ^ { 2 ^ { i + 1 , 2 ^ { j + 1 } } } \left\{\left|\frac{\phi(s, t)}{s t}\right|^{2}+\left|\frac{\phi(-s, t)}{s t}\right|^{2}\right.\right. \\
& \left.\left.+\left|\frac{\phi(s,-t)}{s t}\right|^{2}+\left|\frac{\phi(-s,-t)}{s t}\right|^{2}\right\} d s d t\right\}^{\frac{1}{2}} \\
& \leqq \pi \sum_{0,0}\left\{2^{i+j} \cdot 16 \int_{-\infty,-\infty}^{\infty, \infty} \frac{\sin ^{2}\left(s / 2^{i}\right)}{s^{2}} \frac{\sin ^{2}\left(t / 2^{j}\right)}{t^{2}}|\phi(s, t)|^{2} d s d t\right\}^{\frac{1}{2}}
\end{aligned}
$$




$$
=\pi \sum_{0,0}^{h, k}\left\{2^{i+j} \int_{-\infty,-\infty}^{\infty, \infty}\left|g\left(u+\frac{1}{2^{i}}, v+\frac{1}{2^{j}} ; u-\frac{1}{2^{i}}, v-\frac{1}{2^{j}}\right)\right|^{2} d u d v\right\}^{\frac{1}{2}} .
$$

Now let $\omega\left(g ; \delta^{\prime}, \delta^{\prime \prime}\right)=\sup \left\{\left|g\left(u+\varepsilon^{\prime}, v+\varepsilon^{\prime \prime} ; u-\varepsilon^{\prime}, v-\varepsilon^{\prime \prime}\right)\right|\right.$, where the supremum is taken over $\varepsilon^{\prime}$ and $\varepsilon^{\prime \prime}, 0<\varepsilon^{\prime} \leqq \delta^{\prime}, 0<\varepsilon^{\prime \prime} \leqq \delta^{\prime \prime}$, and over all $u, v$. Under the assumption that $d g(u, v)$ has no mass points, $\omega\left(g ; \delta^{\prime}, \delta^{\prime \prime}\right) \rightarrow 0$ as $\delta^{\prime}, \delta^{\prime \prime} \rightarrow 0$, this convergence being monotonic. Then

$$
\begin{aligned}
& I^{\prime}(m, n) \leqq \pi \sum_{0,0}^{h, k}\left\{\int_{-a-1 .-b-1}^{a+1, b+1} \sum_{1,1}^{2^{i, 2 j}} \mid g\left(u+(2 p-1) / 2^{i}\right.\right. \\
&\left.v+(2 q-1) / 2^{j} ; u+(2 p-3) / 2^{i}, v+(2 q-3) /\left.2^{j}\right|^{2} d u d v\right\}^{\frac{1}{2}} \\
&=2 \pi(V(g))^{\frac{1}{2}}(a+1)(b+1) \sum_{0,0}^{h, k}\left\{\omega\left(g ; 2^{-i}, 2^{-j}\right)\right\}^{\frac{1}{2}} \\
&=C \sum_{0,0}^{h, k}\left\{\omega\left(g ; 2^{-i}, 2^{-j}\right)\right\}^{\frac{1}{2}},
\end{aligned}
$$

since in the range of summation, $g\left(u+2^{-i}, v+2^{-j} ; u-2^{-i}, v-2^{-j}\right)=0$ whenever $(u, v) \notin[a+1, b+1 ;-a-1,-b-1]$ by the assumption that $d g(u, v)$ is identically zero in $\Delta^{\prime \prime}$. Now let $m, n$ run through the sequence $1,2,4, \cdots, 2^{h+1}$ and $1,2,4, \cdots, 2^{k+1}$ respectively. Then, given $\varepsilon>0$, for $h, k$ large enough, there exist integers $h^{\prime}<h$ and $k^{\prime}<k$ such that $\left\{\omega\left(g ; 2^{-i}, 2^{-j}\right)\right\}^{\frac{1}{2}}<\varepsilon, i>h^{\prime}$ and $j>k^{\prime}$. Then

$$
\begin{aligned}
I^{\prime}(m, n) \leqq & C\left\{\sum_{0,0}^{h^{\prime}, k^{\prime}}+\sum_{0 . k^{\prime}+1}^{h^{\prime} . k}+\sum_{h^{\prime}+1,0}^{h, k^{\prime}}+\sum_{h^{\prime}+1, k^{\prime}+1}^{h . k}\right\} \\
< & C^{\prime}\left\{\left(h^{\prime}+1\right)\left(k^{\prime}+1\right)+\left(h^{\prime}+1\right)\left(k-k^{\prime}\right)\right. \\
& \left.+\left(h-h^{\prime}\right)\left(k^{\prime}+1\right)+\varepsilon\left(h-h^{\prime}\right)\left(k-k^{\prime}\right)\right\} \\
= & O(1)\{O(1)+O(h)+O(k)+O(\varepsilon h k)\} \\
= & o(h k)+O(\varepsilon h k) \\
= & o(\log m \log n), m, n \rightarrow \infty
\end{aligned}
$$

since $\varepsilon$ is arbitrary.

To estimate $I^{\prime \prime}(m, n)$, choose $a$ and $b$ so large so that the total variation of $g(u, v)$ on $\Lambda^{\prime \prime}$ does not exceed $\varepsilon$. Then

$$
\begin{aligned}
I^{\prime}(m, n) & =\int_{1,1}^{m, n}\left|\int_{\Delta^{\prime \prime}} \frac{\sin s u}{s} \frac{\sin t v}{t} d g(u, v)\right| d s d t \\
& \leqq \varepsilon \int_{1,1}^{m, n} \frac{d s}{s} \frac{d t}{t}=\varepsilon(\log m \log n) .
\end{aligned}
$$


Thus, if $g(u, v)$ has no mass points, then

since $\varepsilon$ is arbitrary.

$$
\begin{aligned}
I(m, n) & =I^{\prime}(m, n)+I^{\prime \prime}(m, n) \\
& =o(\log m \log n)+\varepsilon(\log m \log n) \\
& =o(\log m \log n), m, n \rightarrow \infty
\end{aligned}
$$

To prove the converse, suppose that $d g(u, v)$ has mass points $\left\{\left(u_{i}, v_{j}\right)\right\}$, $i, j=1,2,3, \cdots$, and let $d_{i j}=g\left(u_{i}^{+}, v_{j}^{+} ; u_{i}^{-}, v_{j}^{-}\right)$be the "jumps" at these points. Let

$$
\begin{aligned}
& g^{\prime}(u, v)=\sum_{u l<u, v j<v}^{\sum} d_{i j} \\
& g^{\prime \prime}(u, v)=g(u, v)-g^{\prime}(u, v) .
\end{aligned}
$$

Then $d g^{\prime \prime}(u, v)$ has no mass points and

$$
\begin{aligned}
I(m, n)= & \int_{1,1}^{m n} \mid \int_{+\infty,-\infty}^{\infty, \infty} \frac{\sin s u}{s} \frac{\sin t v}{t} d g^{\prime}(u, v) \\
& +\int_{-\infty,-\infty}^{\infty, \infty} \frac{\sin s u}{s} \frac{\sin t v}{t} d g^{\prime \prime}(u, v) \mid d s d t \\
= & \int_{1,1}^{m, n}\left|\sum_{i, j} d_{i j} \sin u_{i} \sin v_{j} t\right| \frac{d s}{s} \frac{d t}{t} \\
& +o(\log m \log n), \quad m, m \rightarrow \infty
\end{aligned}
$$

by the proof of the first part. Now

$$
\left|\sum_{i, j} d_{i j} \sin u_{i} s \sin v_{j} t\right|
$$

is an almost periodic function with a positive mean value $M$. Thus

$$
\begin{aligned}
I(m, n) & \rightarrow M \log m \log n+o(\log m \log n) \\
& \neq o(\log m \log n), m, n \rightarrow \infty .
\end{aligned}
$$

This proves the converse of (4).

To prove the equality (3), we apply the Cauchy-Schwarz inequality to the integral in (3), and proceed as before. The converse is also proved in the same manner except that, as already noted, $d g(u, v)$ may have mass points along the coordinate axes in the case of the equality (4). On the other hand, if $d g(u, v)$ has mass points along the coordinate axes, then the equality (3) fails.

\section{References}

[1] S. Bochner, Lectures on Fourier Integrals (Princeton University Press, Princeton (1959)).

[2] S. Bochner and K. Chandarsekharan, Fourier Transforms (Princeton University Press, Princeton (1949)). 
[3] J. A. Clarkson and C. R. Adams, 'On definitions of bounded variation for functions of two variables', Trans. Amer. Math. Soc. 35 (1933), 824-854.

[4] E. W. Hobson, The Theory of Functions of a Real Variable, Vol. II, 2nd edition. (University Press, Cambridge (1926)).

[5] D. Leviatan and L. Lorch, 'The Gibbs phenomenon and Legesgue constants for regular $[J, f(x)]$ means' Acta Math. Hung. 21 (1970), 65-85.

[6] L. Lorch and D. J. Newman, 'The Lebesgue constants for regular Hausdorff methods,' Can, Jour. Math. 13 (1961), 283-291.

[7] E. J. McShane, Integration (Princeton University Press, Princeton (1944)).

[8] W. H. Young, 'On multiple integrals' Proc. Royal Soc. London. (A) 93 (1917), 28-41.

[9] A. Zygmund, Trigonometric Series, 2nd ed., Vol. II (University Press, Cambridge (1959)).

Department of Mathematics

The University of Alberta

Edmonton 7, Canada 